\title{
Do genetics help epidemiologists? Arterial hypertension and cardiovascular events in the light of genetic demiology
}

\author{
Edoardo Casiglia ${ }^{1}$. Valérie Tikhonoff ${ }^{1}$
}

Received: 30 August 2017 / Revised: 6 September 2017 / Accepted: 6 September 2017 / Published online: 23 February 2018

(c) The Japanese Society of Hypertension 2018

Why some subjects are old but remain young in build, whereas other patients are affected by a number of cardiovascular diseases or die prematurely, is a question that remains unanswered. The answer may depend on genetic or environmental factors. Human health, well-being and life expectancy may be partially influenced by lifestyle and partially be genetically determined. Many cardiovascular determinants of premature ageing (such as cigarette smoking) are clearly environmental and others (such as familial dyslipidemia) are only genetic, but the great majority of cardiovascular determinants may have both genetic and environmental origins.

It is hard to distinguish between the two components, and the need for experimental studies is pressing. If the main cause of disease is environmental, evidence-based public health strategies can be carried out to promote healthy aging of the population, while if it is genetic, subjects at risk must receive more resources. Unfortunately, no experimental model in this sense can be carried out in humans. Epidemiology represents the best approximation.

Special populations, such as those due to migration or those secluded in restricted areas, are good epidemiological models. For example, Africans living in Africa and the Amondava Amazon Aborigine appear biologically younger than coeval subjects living in Western societies when cardiovascular risk is measured by an epidemiologist by means of clinical markers, such as blood pressure (BP), serum lipids and uric acid [1], and body weight. When the populations migrate to affluent societies, they acquire a biological age similar to that of the natives, which seems to

These authors contributed equally: Edoardo Casiglia, Valérie Tikhonoff.

Valérie Tikhonoff

edoardo.casiglia@unipd.it

1 Department of Medicine, University of Padova, Padova, Italy highlight the role of environmental factors. However, American blacks who returned to Africa and founded Liberia maintained a Western biological age and are often hypertensive, demonstrating the role of genetic factors. Another model is secluded groups that had limited genetic mixing over the centuries. A good example is people carrying the aproprotein A1-Milan, which came from a strain mutation in the 18th century. These individuals have always lived in strict seclusion and have largely intermarried, demonstrating the key role of the genetic pattern. In other cases, the effects of migration were added to those of seclusion, demonstrating the key role of the environment. This is the case of the Lugalawa people described by Pauletto et al. in Tanzania. These people are composed of two genetically similar populations that were separated and secluded in ancient times and exposed to diverging environmental stimuli, developing a different biological age. A third case is the uncertain balance between genetics and the environment, such as in the Italian secluded valleys described in our ongoing LEOGRA (last evidence of genetic risk factors in the aged) and GOLDEN (growing old with less diseases enhancing neurofuntions) studies, which are aimed at discriminating between genetic-dependent and lifestyle-dependent cardiovascular risk factors.

The maximum life expectancy of humans is approximately 120 years, but humans survive, on average, for 85 years. By preventing diseases and delaying the processes of aging, humans could increase their individual life expectancy and reduce morbidity late in life, leading to healthy aging. Chronic renal disease, chronic obstructive pulmonary disease, arteriosclerosis, increased arterial stiffness, heart failure, and pathological events are the basis of unhealthy aging and can be both the origin and consequence of arterial hypertension. How to prevent these diseases is the main challenge of the 21 st century.

Arterial hypertension represents a typical example of the interaction between genetics and the environment. Other determinants (hypercholesterolemia, hypertriglyceridemia, hyperuricemia being overweight and reduced glucose 

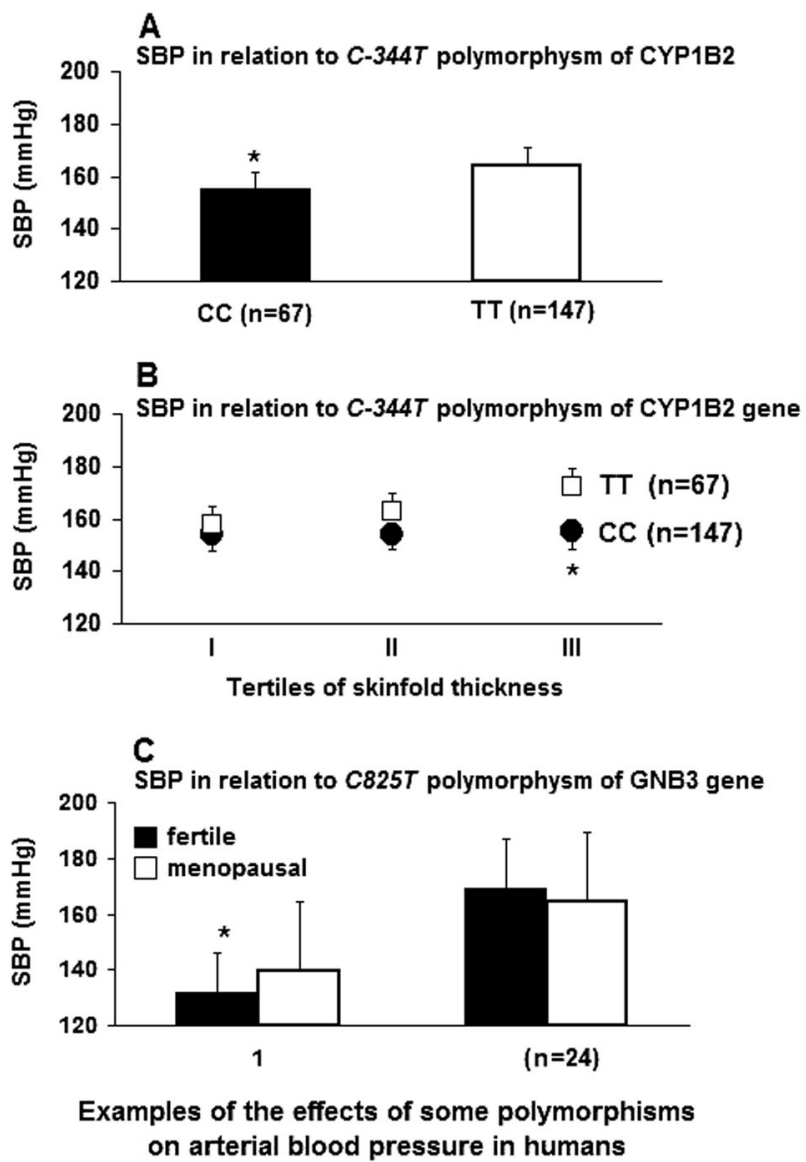

Fig. 1 Examples of the role of genetics in epidemiology. a Systolic blood pressure (SBP) in homozygotes with the $C$-344T polymorphism of the CYP11B2 gene (modified from reference [2]). The TT subjects have higher SBP values compared to the CC patients $(* p<0.05)$. b Only patients homozygous for $C-344 T / T T$ had an increase in SBP with increasing skinfold thickness $(* p<0.05$ vs. III tertile in TT; modified from reference [3]). $\mathbf{c}$ In the patients homozygous for CC in the C825T polymorphism of the GNB3 gene, the SBP adjusted for confounders is higher $(* p<0.01)$ in menopausal than in fertile women. This does not happen in the TT homozygous patients, who have SBP levels that are higher and constant across menopause (they are older than their anagraphic age; modified from reference [4])

tolerance) may also have both genetic and environmental origins. Environmental stimuli activate genetic responses that can modify the course of aging, thereby promoting or suppressing the disease phenotype.

Based on the considerations above, genetics invaded the fields of hypertension and cardiovascular risk in recent years. It has been observed that single-nucleotide polymorphism (SNP) mutations can cause subjects to become more prone to hypertension or to have other risk factors (Fig. 1). For instance, it has been suggested that people carrying the $\mathrm{C}$ allele of the $C-344 T$ polymorphism of the CYP1B2 gene, which encodes for aldosterone synthase, have higher BP levels than those without this trait [2], which are also due to higher of skinfold thickness values that directly correlate with BP [3]. Truncal obesity with excess subcutaneous tissue is also increased in TT subjects with the C825T polymorphism of the GNB3 gene [4]. As adiposity also depends on dietary habits, this is an example of what is called "the echogenetic context". Another cardiovascular case includes heart rate, wherein variability, regulated by the AT1R/A1166C and CYP11B2/C-344T polymorphisms, is dependent on dietary sodium intake [5]. Other examples of the echogenetic context include the intake of recreational beverages [6], which can influence cardiovascular risk depending on genetic patterns [7, 8], and by cognitive/mental patterns, which are, in part, genetically determined and can be both the consequence and cause of cardiovascular risk [9-11].

It is undisputed that essential arterial hypertension is the consequence of a genetic mosaic, which makes the genomewide $(\mathrm{GW})$ scan particularly interesting. The secret is to be able to build a picture that represents the truth rather than our mental image. This is also true for cardiovascular diseases, such as coronary artery disease.

Recently, analysis of SNPs has been questioned and the GW scan has become more common. This technique allows association studies aimed at finding links (also unexpected) between genetic traits and a disease. The advantage of a GW scan is that it leads to a large amount of putative associations; its disadvantage is that the amount of data generated is often excessive and can lead to misleading conclusions. The forcefulness of the GW results depends on the signature at the bottom of the scientific article.

In the paper shown in the present issue regarding this topic [12], Wirtwein et al., using a Genetic Risk Score (GRS19) identified a well-defined series of SNPs (namely, the rs1746048/CXCL12, rs9349379/PHACTR1, rs 17465637/MIA3, rs3798220/LPA, rs9818870/MRAS, $r s 17114036 / \mathrm{PPAP} 2 \mathrm{~B}, \quad r s 12413409 / \mathrm{CNNM} 2, \quad r s 6725887 /$ WDR12, $\quad r s 9982601 / \mathrm{KCNE} 2, \quad r s 12190287 / \mathrm{TCF} 21$, rs 17609940/ANKS1A, rs3825807/ADAMTS7, rs4977574/ Chr9p21, rs1122608/LDLR, rs11206510/PCSK9, rs216172/SMG6, rs 12936587/RALI, rs11556924/ZC3HC1 and $r s 2259816 / \mathrm{HNF} 1 \mathrm{a})$ that were good candidates for major adverse cardiovascular events in relation to nighttime BP. In brief, subjects with normal day-time and elevated night-time BP had a higher GRS19 than those with normal day-time and normal night-time BP, and after adjusting for cardiovascular risk factors, GRS19 determined night-time systolic BP. The obvious conclusion is that a high night-time systolic BP is genetically determined and is in turn a determinant of cardiovascular events in patients with coronary artery disease. In other words, the 19 SNPs listed above indirectly determine the cardiovascular risk. This is what we can expect from a GW scan applied to the field of arterial hypertension: a prediction based on a significant interaction between two or more factors (in the present case, the genetic pattern, night-time BP and pre- 
existing coronary disease). Demanding that the analysis of SNPs or even a GW scan provide a complete picture of the pathogenetic origin of hypertension would not be possible.

Unfortunately, the GW scan is quite expensive and is not suitable for everyone.

The final question is: did the GW scan and genetics, in general, add something to the expertize of epidemiologists dealing with arterial hypertension and cardiovascular risk [13]? Is there a future for genetics in epidemiology? Normalization of hypertension is obtained only in $6-25 \%$ of cases. This derives from five major components: (1) a limited number of hypertensive causes are usually identified, (2) not all identified hypertensive causes are treated, (3) the few patients who receive medication are treated incompletely, (4) the targets suggested by international guidelines (often contrasting) are rarely reached, and (5) the genesis of hypertension remains unknown, making a pathogenetic therapeutic approach impossible. All contributions that increase knowledge in this field can contribute, in turn, to better treatment and have positive clinical repercussions. In this sense, any intrusion of genetics in the field of epidemiology should be considered favorably.

\section{Compliance with ethical standards}

Conflict of interest The authors declare that they have no conflict of interest.

\section{References}

1. Mazza A, Zamboni S, Rizzato E, Pessina AC, Tikhonoff V, Schiavon L, Casiglia A. Serum uric acid shows a J-shaped trend with coronary mortality in non-insulin-dependent diabetic elderly people. The CArdiovascular STudy in the ELderly (CASTEL). Acta Diabetol. 2007;44:99-105.

2. Casiglia E, Tikhonoff V, Mazza A, Rynkiewicz A, Limon J, Caffi S, Guglielmi F, Martini B, Basso G, Winnicki M, Pessina AC, Somers VK. C-344T polymorphis of the aldosterone synthase gene and blood pressure in the elderly: a population-based study. $\mathrm{J}$ Hypertens. 2005;23:1991-6.
3. Casiglia E, Tikhonoff V, Schiavon L, Guglielmi F, Pagnin E, Bascelli A, Basso G, Mazza A, Martini B, Bolzon M, Guidotti F, Caffi S, Rizzato E, Pessina AC. Skinfold thickness and blood pressure across $C-344 T$ polymorphism of CYP11B2 gene. J Hypertens. 2007;25:1828-33.

4. Casiglia E, Tikhonoff V, Caffi S, Martini B, Guidotti F, Bolzon M, Bascelli A, D'Este D, Mazza A, Pessina AC. Effects of the C825T polymorphism of the GNB3 gene on body adiposity and blood pressure in fertile and menopausal women: a populationbased study. J Hypertens. 2008;26:238-43.

5. Stolarz K, Staessen JA, Kawecka-Jaszcz K, Brand E, Bianchi G, Kuznetsova T, Tikhonoff V, Thijs L, Reineke T, Babeanu S, Casiglia E, Fagard R, Filipovsky J, Peleska J, Nikitin Y, StruijkerBoudier H, Grodzicki T. Genetic variation in CYP11B2 and AT1R influences heart rate variability conditional on sodium excretion. Hypertension. 2004;44:156-62.

6. Casiglia E, Paleari CD, Petucco S, Bongiovì S, Colangeli G, Baccillieri MS, Pavan L, Pernice M, Pessina AC. Haemodynamic effects of coffee and purified caffeine in normal volunteers: a placebo-controlled clinical study. J Human Hypertens. 1992;6:95-9.

7. Palatini P, Benetti E, Mos L, Garavelli G, Mazzer A, Cozzio S, Fania C, Casiglia E. Association of coffee consumption and CYP1A2 polymorphism with risk of impaired fasting glucose in hypertensive patients. Eur J Epidemiol. 2015;30:209-17.

8. Casiglia E, Tikhonoff V, Albertini F, Montagnana M, Danese E, Mazza A, Favaro J, Finatti F, Benati M, DalMaso L, Gasparotti F, Spinella P, Palatini P. Effects of caffeine and coffee on incident heart failure in general population. role of the CYP1A2 -163C $>$ A polymorphism. WIRR. 2017;5:42-48.

9. Tikhonoff V, Casiglia E, Guidotti F, Giordano N, Martini B, Mazza A, Spinella P, Palatini P. Body fat and the cognitive pattern: a population-based study. Obesity. 2015;23:1502-10.

10. Tikhonoff V, Hardy R, Deanfield J, Friberg P, Kuh D, Muniz G, Pariante CM, Hotopf M, Richards M. Symptoms of anxiety and depression across adulthood and blood pressure in latemiddle age: the 1946 British birth cohort. J Hypertens. 2014;32:1590-9.

11. Tikhonoff V, Hardy R, Deanfield J, Friberg P, Muniz G, Kuh D, Pariante CM, Hotopf M, Richards M. The relationship between affective symptoms and hypertension-role of the labelling effect: the 1946 British birth cohort. Open Heart. 2016;3:e000341.

12. Wirtwein $M$, Melander $O$, Sjőgren $M$, Hoffmann $M$, Narkiewicz K, Gruchala M, Sobiczewski W Genetic risk factors influence nighttime blood pressure and related cardiovascular complications in patients with coronary heart disease. Hypert Res. 2018;41:53-9.

13. Casiglia E, Tikhonoff V. Old-style epidemiology and epigeneticdemiology. Hypert Res. 2015;38:380-1. 